\title{
RELACIÓN ENTRE LA ENSEÑANZA DE LAS MATEMÁTICAS Y LAS CIENCIAS BIOLÓGICAS
}

\section{RELATION BETWEEN MATHEMATICS AND BIOLOGICAL SCIENCES TEACHING}

\author{
DE LA OSSA, V. LUZ STELLA ${ }^{2^{*}}$ M.Sc., DE LA OSSA-LACAYO, ALEJANDRO ${ }^{2^{* *}}$ \\ Ecólogo. \\ ${ }^{1 *}$ Profesora Universidad de Sucre, Colombia. Facultad de Educación y \\ Ciencias. ${ }^{2}$ Fundación George Dahl, Colombia. \\ *Correspondencia: luzdelaossa2000@yahoo.com.mx
}

Recibido: 03-02-2010; Aceptado: 08-03-2010

\section{Resumen}

El presente trabajo de manera crítica aborda las relaciones existentes entre las matemáticas y las ciencias biológicas, enfatizando en la importancia y necesidad reciproca que tienen ambas ramas del saber como parte fundamental de la investigación científica moderna. Plantea la necesidad de llevar a cabo cambios en los modelos de enseñanza de estas dos áreas en la educación superior actual. Muestra de forma diferencial el enfoque de las matemáticas al servicio de la realidad y de la realidad al servicio de las matemáticas. Al tiempo que ratifica el valor actual de la modelación como vía de explicación de los fenómenos naturales.

Palabras clave: matemáticas, ciencias biológicas, enseñanza superior, importancia investigativa.

\section{Abstract}

The present work is a critical approach of the existent relationships between the mathematics and the biological sciences, emphasizing in the importance and reciprocity that have both branches of the knowledge as fundamental part of the modern scientific research. It emphasizes the necessity to carry out changes in the models of teaching of these two areas in the current superior education. It shows in a differential way the focus from the mathematics to the service of the reality and the reality to the service of the mathematics. At the time that ratifies the current value of the mathematical models like road of explanation of natural phenomenons. 
Key words: mathematics, biological sciences, higher education, research importance.

\section{Introducción}

Existe actualmente, en lo referente a la educación un panorama que enmarca el avance científico y tecnológico de desarrollo continuo que requiere mayores esfuerzos para alcanzar una formación integral del hombre futuro, se observa una elevada demanda de este tipo de educación al tiempo que se muestra una gran tendencia a la diversificación de este tipo de formación educativa, con una mayor toma de conciencia por la calidad del proceso en mención , y en función del desarrollo sociocultural, económico, ambiental y participativo que requiere la construcción de futuro en consonancia con la necesidad de formar activamente según las competencias, nuevos conocimientos e ideales que requiere el futuro (UNESCO, 1998).

La educación debe proporcionar los mecanismos de actualización continua de los conocimientos adquiridos y visualizar de forma práctica la integración activa entre las diferentes disciplinas como único mecanismo de comprensión de los fenómenos naturales, debe así mismo visualizarse en torno a cuatro tipos de aprendizaje: aprender a conocer, aprender a hacer, aprender a convivir y aprender a ser. En este último punto se enfatiza en que cada persona debe ser estimulada educativamente para obtener y mantener un pensamiento autónomo y crítico que le señale su quehacer en cada una de las situaciones de vida en las que se encuentre, y que estos cuatro tipos de aprendizaje son fundamentales en la enseñanza de las ciencias, en especial cuando se hace referencia a la educación superior, ya que más que la cantidad de conocimientos que se puedan almacenar, la formación de actitudes y la capacidad de respuesta a estímulos generados por problemas a dilucidar deben ser la mayor preocupación en la formación científica (Di PRISCO, 2001).

En tal sentido, la enseñanza de las ciencias, hablando de forma muy particular, y tomando como referencia lo expresado por DI PRISCO (2001), debe estar orientada a desarrollar la capacidad de enfrentar problemas novedosos aplicando los conocimientos adquiridos y buscando conocimientos que puedan ser de utilidad o produciéndolos a través de la investigación, sin reducir el concepto, entendiendo que se hace necesario que se cuente con una base sólida de conocimientos fundamentales, con una estructura racional y crítica de sus competencias para seleccionar, apropiar, transformar y aplicar información necesaria que ayude a comprender el significado de las cosas per se.

Por su parte De VENANZI (1987) indica que los objetivos misionales de una Universidad: docencia, extensión e investigación, deberían ser constantemente enriquecidos, de forma oportuna con fundamento en la realidad problemática 
del entorno mediante los resultados que se deriven de las investigaciones que están obligadas a realizar las universidades y sus investigadores; los proyectos de investigación en tal sentido son la base de la renovación y crecimiento académico del profesorado universitario y por lo tanto muestran su importancia en la docencia y la extensión que el claustro adelante y son quienes posicionan su valor ante la comunidad a la cual se debe.

En el presente trabajo se discutirá la importancia de una formación integral y activa entre las matemáticas y las ciencia biológicas como parte importante en la adquisición de competencias para los nuevos profesionales y en especial se hará énfasis en la necesidad justificada de esta relación para el abordaje de la investigación, entendiendo que es necesario en cualquier campo del desarrollo de una profesión la resolución de problemas desde una perspectiva lógica y critica que permita el avance del conocimiento y el bienestar social.

\section{Enfoque de las matemáticas y su relación con las ciencias biológicas}

Teniendo en cuenta lo planteado por CAÑADA (1991), la pregunta simple que debe hacerse y sobre la cual gravita el dilema de la enseñanza de las matemáticas en los programas de formación universitaria en ciencias biológicas, es: ¿el objeto de las matemáticas es explicar fenómenos naturales o es una disciplina cuyo propósito es el desarrollo armónico de cuestiones abstractas, sin aplicación real de sus conclusiones?

En sentido argumentativo, podría decirse que históricamente las matemáticas tienen origen en el intento de explicar racionalmente fenómenos concretos de otras ciencias, como: física. Ingeniería, astronomía y biología, por ejemplo. En todos los casos prima la curiosidad científica para tratar de entender las cuestiones relacionadas con el problema y sus posibles relaciones con otros fenómenos. No obstante, se tienen abundantes ejemplos de teorías que nacieron de manera abstracta y que posteriormente han sido de gran utilidad en el estudio de la naturaleza (CAÑADA, 1991).

En cuanto a la enseñanza de las matemáticas en los programas de ciencias biológicas se ha presentado un reciente gran cambio; se pensaba que una buena formación podría ayudar al biólogo o profesional de ciencias afines a entender algunas leyes y principios de la biología, teniendo como fundamento entre otros temas, los siguientes: teoría de conjuntos, funciones, derivación, sistemas de ecuaciones y actualmente principios computacionales y manejo de algunos paquetes de análisis (CARDÚZ, 1972). Sin embargo, la situación real es radicalmente diferente cuando se hace referencia a investigación, puesto que la mayoría de los problemas planteados en el campo de la biología, crean la necesidad de descubrir nuevas teorías matemáticas 0 de potenciar 0 desarrollar las ya existentes (CAÑADA, 1991), con el propósito de establecer una modelación particular y novedosa que se ajuste a la realidad, en la cual se 
puedan explicar innumerables variables influyentes en el problema y su relaciones entre sí.

\section{Realidad al servicio de las matemáticas}

Ya se manifestaba en 1913 que entre los muchos cambios de la educación matemática suscitado durante los últimos 20 años, persistía un elemento constante, el deseo de relacionar la matemática con la realidad, mostrarla como un cuerpo vivo de pensamiento que influye y puede influenciar la vida humana en multitud de puntos (CARSON,1913 apud HOWSON y WILSON, 1991); desde entonces ya existía una seria preocupación por la aplicación práctica de las matemáticas y su relación con la ciencias de la vida y sus interrogantes, aunque históricamente la enseñanza de las matemáticas siempre estuvo caracterizada por ser un componente vocacional que con la institucionalización a nivel de escuelas y universidades inició su distorsión, lo cual se fundamenta en razones complejas, como lo señalan HOWSON y WILSON (1991) al considerar que existe un serio problema de enseñanza de aplicaciones y de uso de ejemplos reales que aún está por resolver. A pesar de los avances que se tienen en la actualidad, todavía persiste en muchos claustros y programas de formación académica superior la poca aplicación real de los contenidos matemáticos en las áreas de las ciencias, he ahí el dilema a resolver de forma acertadamente práctica: ¿Es posible asegurar que todas las situaciones que se presenten a los alumnos sean realmente prácticas y reales o se debería intentarse que lo fueran?

Muchas situaciones que no son prácticas ni reales, son una herramienta en la cual se acepta que existe la actitud de resolver un problema como parte de la estructuración del pensamiento creativo; los ejemplos artificiales no necesariamente son malos, ya que el arte de aprender a aplicar las matemáticas puede cultivarse con situaciones que no son de la vida real (HOWSON y WILSON, 1991), pero que incentivan y refuerzan la capacidad para deducir, interpretar, aplicar y resolver, lo que en esencia es una importante competencia.

Existen también los ejemplos vocacionales como el que persiste en el uso de libros guías específicos o asociados a particulares tipos de empresas productivas, en muchos casos típicamente regional, circunscritos a un panorama de demanda profesional direccionado que se inserta en un mundo global y en permanente cambio, podría entonces conceptuarse, coincidiendo con HOWSON y WILSON (1991), que se está preparando un estudiante dentro de una realidad temporal estática y con arraigo en el pasado, sin la necesarias connotaciones que el futuro plantea para las nuevas generaciones y la resolución de los problemas inherentes a su época. No obstante, siempre deberá existir la necesidad de aplicaciones que se sustenten en las necesidades de la sociedad actual y que en muchos casos son relativamente 
estables, pero que deben permitir visionar los posibles cambios que se deben presentar adicionalmente; sin que lo local sea menos importante, se debe transcender hacia lo nacional y lo global, como vía lógica de la evolución social moderna.

CARSON (1913) apud HOWSON y WILSON (op cit) señala de manera textual, que: la esencia de la realidad se encuentra en preceptos y conceptos reconocibles y es, por lo tanto, una función del individuo y la época; lo que es real para mí no es necesariamente real para otro, y mucho de lo que era real para mí en la infancia ya no lo es. Es el profesor quien debe determinar las realidades de sus alumnos... También debe encontrar necesario ampliar sus esferas de realidad, pero debe evitar la confusión entre un nombre y una cosa.

\section{Matemáticas al servicio de la realidad}

Cuando se utilizan las matemáticas para resolver problemas reales presentes en la sociedad y su entorno, se tiene un panorama diferente, en donde se requiere no solo por su importancia social sino porque implican la aplicación de variadas técnicas matemáticas, acervo de conocimientos diversos sobre el tema especifico y conexo, al tiempo que ofrece la posibilidad de extensión en diferentes vías de aplicación (HOWSON y WILSON,1991), como sucede cuando se trata de establecer la movilidad de una población animal dada dentro de un área en función del tiempo y de las condiciones ambientales, cuando se investigan diversas patologías en función de las condiciones socioeconómicas, cuando se hacen análisis genéticos de poblaciones y sus variaciones en función del tiempo, cuando se busca comprender las causas y consecuencias de vertimientos tóxicos en cuerpos de agua. Entonces aquí las matemáticas son instrumentales, son la llave conectora entre las diferentes actuaciones, conceptualizaciones y postulados interdisciplinares que se requiere para un abordaje complejo del problema y el planteamiento de sus posibles soluciones en función espacio temporal.

En cuanto a la enseñanza de este tipo de matemáticas los problemas que surgen se relacionan con la capacidad del alumno para comprender la realidad que se establece en la situación, capacidad creativa, intelectual y social que satisfaga las diferencias individuales y la integración de actividades diversas en grupo, capacidad para obrar con iniciativa propia y preparación básica para acceder razonablemente a plantear soluciones; anotando que muchos de estos inconvenientes son también parte de la estructura del profesor, ya que en muchos casos carece de la información necesaria para comprender académicamente el problema en el cual deberá trabajar o enseñar a resolver, limitando el proceso de comprensión y análisis que se necesita en conjunto para establecer los modelos. 
Los modelos reales que se requieren implican tener la capacidad de moverse en diferentes niveles, es necesario considerar múltiples opciones de modelos, combinarlos, abstraer principios y procedimientos incluidos en ellos, que son la base conceptual de las matemáticas aplicadas para tener la confianza necesaria en la aplicación (HOWSON y WILSON, 1991).

\section{Explicaciones biológicas y modelos matemáticos}

Las matemáticas han tenido una larga intervención en las ciencias biológicas y la sinergia entre ambas ha enriquecido los logros de ambas ciencias por muchos años (HASTINGS y PALMER, 2003), y se espera que para las próximas décadas esta relación sea mucho más fructífera (COHEN, 2004; MAY, 2004). Se trata de una relación, que como lo señala KARI (1997) posee una alta interdisciplinaridad, que como en el caso de los modernos estudios de genética queda evidentemente establecida desde los planteamientos estocásticos y las aplicaciones estadísticas que son requeridas para llegar a explicaciones reales en este campo de la biología. De allí la importancia de mantener un especial cuidado con las estrategias necesarias para la enseñanza de las matemáticas en la formación de los estudiantes universitarios de las ciencias biológicas (JUNGCK, 1997; PÉREZ et al., 2006).

Haciendo referencia a la aplicación de las matemáticas en ecología, importante rama de las ciencias biológicas y disciplina de síntesis por excelencia, cuando se busca modelar se tienen tres niveles jerárquicos bien definidos: población, comunidad y ecosistema, en el segundo nivel jerárquico fue donde el modelaje matemáticos tuvo su inicio, expresado por MALTHUS (1978) para entender el crecimiento poblacional, este modelo muy importante, es simple y se basa en una ecuación diferencial; otro modelo bastante conocido en ecología fue planteado por LOTKA (1925) y VOLTERRA (1926), hace referencia a la relación presa-depredador, las ecuaciones planteadas describen cambios oscilatorios en dos poblaciones que interactúan en función del tiempo (ANGELINI, 2000). Cuando se hace referencia a ecosistema la complejidad de los análisis es mucho mayor, debe usarse un análisis de sistemas para su comprensión, esto permite que los fenómenos complejos sean divididos en partes elementales o comunes posibilitando la aplicación de métodos cuantitativos (von BERTALANFFY, 1977), lo cual también es aplicable cuando se trata de comunidades.

En general, como lo indica MILLÁN-GASCA (1996) el concepto moderno de investigación en matemática aplicada se fundamenta en un modo característico de entender la relación entre las matemáticas y la realidad centrada en la noción de modelo y la metodología de la modelización, al respecto ANGELINI (2000) conceptúa que independientemente del tipo de problema biológico que se pretenda analizar la confección del un modelo incluye las siguientes fases: 
1. Reconocer o delimitar el sistema de interés, expresándolo de preferencia a través de un diagrama conceptual, con el fin de visualizar las interacciones entre los componentes, y su posible análisis unitario o fraccionado, según el caso (población, comunidad o ecosistema)

2. Escoger la estructura cuantitativa general $<<$ ecuaciones diferenciales, matriz algebraica, modelo de compartimiento, etc. $>$ seleccionando la unidad de tiempo para realizar una simulación.

3. Observar la validez del modelo, con el fin de confirmar sí es adecuado para satisfacer los objetivos propuestos, lo cual incluye: análisis de sensibilidad respecto de los parámetros evaluados, en qué medida estos modifican los resultados del modelo; verificación de la calibración, descubrir que valores minimizan la diferencia entre los valores observados y los calculados por el modelo; validación, que es la prueba que se debe hacer para saber si los parámetros son validos para otros periodos dentro del mismo sistema analizado.

\section{Sobre la enseñanza de las ciencias}

Tomando como base los planteamientos de GONZÁLEZ-ARROYO (1988), sobre la función social de la enseñanza de las ciencias, puede decirse, que respecto de las matemáticas y su relación con las ciencias naturales se cometen variados errores didácticos, las prácticas pueden ser monótonas y repetitivas, con falta de sensibilidad en las pruebas y exámenes que se llevan a cabo, aspectos que generan miedo y no en pocos casos rechazo por parte del estudiante que no encuentra amena ni aplicable las matemáticas y menos aún cuando estás tratan de ser la vía de explicación científica de las llamadas ciencias naturales.

Existe por lo general un distanciamiento de los métodos didácticos y racionales de la enseñanza aprendizaje definidos modernamente por la pedagogía. No se puede pensar que la enseñanza de las ciencias tiene que ver solo con lo pedagógico o como si fuera un compendio epistemológico simple o si se tratara de un proceso relacionado con la psicología del aprendizaje, debe avocarse como un diálogo respetuoso de saberes científicos en donde entran en juego lo aprendido por el estudiante, el saber científico acumulado y sistematizado históricamente, la realidad del entorno y las necesidades sociales (GONZÁLEZARROYO, 1988). Así podría entonces pensarse en una renovación del proceso de enseñanza mucho más funcional y clara, que sin duda favorecería la relación entre matemáticas y ciencias biológicas.

De forma reflexiva, y hablando del origen de las matemáticas, se sabe que su nacimiento buscó cubrir las necesidades de contar y medir, en Grecia se 
convierte en una disciplina formal, en donde prima el método demostrativo como base de su estructura y es esté método el mayor aporte a la ciencia en la construcción de otras disciplinas, es allí en Grecia, en donde también nace la idea de introducir las matemáticas en el análisis y resolución de problemas inherentes a otras ciencias, se atribuye a Aristóteles la aplicación de las matemáticas a la mecánica como primer ejemplo de matematización necesaria de una ciencia (HERNÁNDEZ-GUARCH, 2000).

Entonces, desde una perspectiva fundamental se puede afirmar que las abstracciones matemáticas tienen un relevante componente histórico que demuestra la importancia de estas en la resolución de problemas, no es algo novedoso y por lo tanto no pueden verse como un cuerpo abstracto y menos aún repetitivo, porque su función es dinámica y puede variar de acuerdo con la necesidad que se pretenda descubrir, explicar o solucionar. CROMBIE (1975) señala que la idea de la demostración matemática fue, en efecto, el gran descubrimiento de los griegos en la historia de la ciencia, y la base no sólo de sus importantes contribuciones a la misma matemática y a las ciencias físicas, como la astronomía y la óptica geométrica, sino también la base de gran parte de su biología y medicina. Es la idea de la explicación racional, que un hecho concreto es explicado cuando puede ser deducido de un principio más general.

VELASCO-HERNÁNDEZ (2000) expresa que desde mediados del siglo pasado la biología se ha colocado en un lugar prominente del desarrollo científico mundial y que a partir de principios del siglo XXI se dará un gran desarrollo de esta ciencia, que se fundamentará en la aplicación de métodos cuantitativos para la descripción, explicación, análisis y predicción de procesos biológicos. De forma irreverente, pero cierta por demás, dice que: "La época de los biólogos "cuentapatas" ha quedado atrás; ahora prácticamente cualquier área de la biología requiere de la aplicación de al menos, métodos estadísticos que permitan dilucidar causas y efectos en los fenómenos estudiados".

Por otro lado MAY (2004), acertadamente señala que las ciencias físicas, la matemática teórica y la investigación experimental siempre han marchado juntas, pero que las matemáticas han sido menos intrusivas en las ciencias de la vida. No obstante, las posibilidades actuales son mayores y un ejemplo de esto se da en la fuerte introducción metodológica de la estadística en los diseños experimentales, bioinformática, modelos evolutivos, ecología y epidemiología, que si bien aportan valiosas explicaciones también han estado sujetas a un abuso de su aplicación.

\section{Algunos problemas docentes en la enseñanza de las matemáticas}

Por lo general los docentes vinculados a la enseñanza universitaria de las matemáticas en los programas de ciencias biológicas o relacionados poseen formación como especialistas en el área pura o aplicada, algunos pocos cuentan con maestría y son significativamente menos los que poseen título de 
doctorado, se requieren modificaciones estructurales profundas que promuevan una nueva actitud de los profesores, que les permita abandonar el papel de trasmisores de conocimiento y que ayude a los estudiantes a construir su propio conocimiento. La actitud tradicional y axiomática de la enseñanza de las matemáticas, en este sentido, que se basa en la exposición de contenidos en donde los términos son definidos, los axiomas aceptados, los teoremas demostrados, los ejemplos y problemas reducidos a un mínimo necesario, apenas para ilustrar los conceptos no es constructiva, se debe buscar la fundamentación teórica en las propuestas curriculares enfatizando en la resolución de problemas como metodología preferencial (CURY y BAZZO, 2001).

Nos enfrentamos a una enseñanza tradicional de las matemáticas, en donde el carácter libresco y fotocopiesco predomina, en donde la preocupación del estudiante por la formulas, reglas y tablas supera con creces su aptitud de aprendizaje, en donde, por lo general, la mayor influencia sobre el alumno la hacen los medios de comunicación y las charlas informales, y en donde no se desarrolla una actitud crítica, así poco puede esperarse en el desarrollo de las ciencias biológicas, dado el desarraigo existentes en ambas direcciones y la necesidad que existe de unir estos dos extremos, aparentemente tan alejados pero realmente partes continuas del mismo conjunto.

De forma crítica VASCONCELOS (1996) describe el perfil de los profesores de matemáticas, no tan disímil del de los profesores de biología: o son profesores o son investigadores, existiendo, especialmente en el área de las ciencias biológicas aquellos que tratan de conciliar la existencia del profesor investigador, pero que por preocupaciones personales de tipo laboral o por exceso de trabajo externo a veces no pueden desarrollar toda su capacidad, perjudicando una de las dos actividades o sacrificándolas todas (CURY y BAZZO, 2001).

Tomando a CURY y BAZZO (2001) se pueden ejemplarizar algunas acciones prioritarias que deberían ayudar a subsanar la distancia entre la enseñanza de las matemáticas y su valor de uso:

$\checkmark$ Desarrollo de las matemáticas como un mecanismo para comprender y analizar datos cronológicos, a través de un cuestionamiento de las causas y consecuencias de cada hallazgo o invento, tratando de explicar el contexto histórico y social que favoreció su creación, expansión y uso.

$\checkmark$ Los modelos matemáticos en las ciencias deben ser considerados como posibilidades, que no deben perder en la construcción su esencia, que no pueden simplificar la estructura de la explicación porque sin estas precauciones se podría llegar a afectar la sociedad. 
$\checkmark$ El uso de la estadística debe incluir la presentación de variables que relacionen sus pruebas con la sociedad y el medio ambiente, cuidando de no ser un instrumento de maquillaje para los datos y sus implicaciones.

$\checkmark$ Entender que las cuestiones éticas y políticas se relacionan con las matemáticas y su enseñanza, dando el debate necesario para mostrar que las matemáticas se usan como factor de exclusión por aquellos que detentan el conocimiento o son agentes del Estado, como sucede en los problemas relacionados con el género, canasta familiar, empleo, etc.

\section{Conclusiones}

Teniendo en cuenta los textos revisados (GONZÁLEZ-ARROYO, 1988; HOWSON y WILSON, 1991; MILLÁN-GASCA, 1996; VASCONCELOS, 1996; JUNGCK, 1997; KARI ,1997; ANGELINI, 2000; HERNÁNDEZ-GUARCH, 2000; VELASCO-HERNÁNDEZ, 2000; CURY y BAZZO, 2001; HASTINGS y PALMER, 2003; COHEN, 2004; MAY, 2004; PÉREZ et al., 2006) se plantea, que:

1. La enseñanza de las matemáticas tradicionalmente se circunscribe a la adquisición de aptitudes por parte del alumno, se descuidan los procesos, dejando de lado la posibilidad de crear interés por la generalización social del valor de las matemáticas en todas las escalas de la vida y su valor de aplicación en la resolución de problemas.

2. La realidad al servicio de las matemáticas deberá ser cuidadosamente examinada para que en cada caso concreto se permita avanzar en una formación multidisciplinar en donde los conceptos de análisis y la capacidad de abstracción ayuden a fundamentar la comprensión de los procesos biológicos, sociales o ambientales de una manera más expedita y motivacional.

3. Las matemáticas al servicio de la realidad deben ser de aplicación multidisciplinar para que se puedan establecer los modelos de mejor opción y de mayor seguridad en su utilización, razón por la cuales deberá ser un tema compartido, abordado en grupo y teniendo en cuenta los diferentes postulados de cada una de las disciplinas involucradas en el proceso de análisis de la problemática, en donde el hilo conector entre las partes sea el proceso de modelaje matemático.

4. No se debe perder de vista el valor fundamental de las matemáticas en su proceso de enseñanza aprendizaje, es claro que su valor demostrativo y su capacidad creativa permiten explicar de forma racional y lógica los fenómenos naturales, así mismo tienen expreso valor de 
utilización en la explicación y solución que requieren los problemas de las ciencias biológicas.

5. Ante todo se hace necesario impulsar la enseñanza de matemáticas que permitan dar soluciones a los problemas modernos de las ciencias biológicas, buscando ofrecer una formación científica sólida de probada interdisciplinariedad en los cursos académicos universitarios... ni el matemático aislado, ni el biólogo solitario, ni el alumno repetitivo y acrítico: clave de un proceso fructífero.

\section{Referencias}

ANGELINI, R. 2000. Ecologia e modelagem matemática. Ciênc. Biol. Ambient. $2(2): 225-242$.

CAÑADA, A. 1991. De las matemáticas para biólogos a la biología matemática: Un punto de vista particular a través del Análisis Matemático y la Dinámica de Poblaciones. Disponible: http://www.ugr.es/ acanada/investigacion/jaen99.pdf Consultado 3-01-2010.

CARDÚS, D. 1972. Introducción a las Matemáticas para Médicos y Biólogos, Editorial Vicens Vives. España.

COHEN, J.E. 2004. Mathematics Is Biology's Next Microscope, Only Better; Biology Is Mathematics' Next Physics, Only Better. Plos Biology 2 (12):20172023.

CROMBIE, A. C. 1976. Historia de las ciencias: de San Agustín a Galileo. Alianza Universidad. Madrid, España.

CURY, N. H.; BAZZO, W.A. 2001.Formação Crítica em Matemática: Uma Questão Curricular? Bolema 14(16):29-47.

De VENANZI, F. 1987. Investigación y docencia en la universidad. Interciencia 12:221-225.

Di PRISCO C. 2001. La enseñanza de la ciencia y los cuatro pilares de la educación. Interciencia 26 (12):581-586.

GONZÁLEZ- ARROYO, M. 1988. A função social do ensino de ciências. Em Aberto 7(40):3-11.

HASTINGS, A.; PALMER, M.A. 2003. A bright future for biologists and mathematicians? Science 299:2003-2004. 
HERNÁNDEZ-GUARCH, F. 2000. Matemáticas y evolución. Seminario «OROTAVA» de historia de la ciencia - Año V. Disponible en: http://www.gobcan.es/educacion/3/Usrn/fundoro/archivos\%20adjuntos/publicaci ones/actas/actas $45 \mathrm{pdf} /$ Act.IV-V C015 txi w.pdf

Consultado: 3-02-2010.

HOWSON, G.; WILSON, B. 1991. La enseñanza de contenidos específicos en matemáticas. Comunicación, Lenguaje y Educación 11-12:121-137.

JUNGCK, J.R. 1997. Ten equations that changeg biology: mathematics in problem-solving biology curricula. Bioscene 23(1):11-21.

KARI, L. 1997. DNA Computing: arrival of biological mathematics. The mathematical intelligencer 19 (2):9-22.

LOTKA, A. J. 1925. Elements of physical biology. Baltimore, Williams and Wilkins. (Reprinted with corrections and bibliography as Elements of Mathematical Biology. Nova York. 1956).

MALTHUS, T. R. 1798. An essay on the principle of population, as it affects the future improvement of society, with remarks on the speculations of Mrs. Godwin, M. Condorcet and others writers. London, U.K, J. Johnson, V IX.

MAY, R.M. 2004. Uses and Abuses of Mathematics in Biology. Science 303 (5659):790-793.

MILLÁN-GASCA, .1996. "El ideal de la matematización." La aplicación de las matemáticas a las ciencias biológicas, humanas y sociales. Arbor 606:79-102.

PÉREZ, J.E.; PÉREZ, I.; OJEDA, G. 2006. La enseñanza de las ciencias biológicas en la Universidad. Saber 18 (2):234-240.

UNESCO 1998. Marco de acción prioritaria para el cambio y el desarrollo de la educación superior.

http://www.unesco.org/education/educprog/wche/declaration spa.htm.

Consultado: 21-10-2008.

VASCONCELOS, M.L.M.C. 1996. A formação do professor de terceiro grau. Ed. Pioneira. São Paulo, Brasil.

VELASCO-HERNÁNDEZ, J.X. 2000. Biología y matemáticas: la pareja esencial.

Disponible: http://www.sectormatematica.cl/ciencias\%20naturales/biologia\%20matematica. $\underline{\mathrm{pdf}}$ 
Consultado: 2010. 05-02-2010.

VOLTERRA, V. 1926. Fluctuations in the abundance of a species considered mathematically. Nature 118:558-560.

VON BERTALANFFY, L. 1977. Teoria geral dos sistemas. Rio de Janeiro, Ed. Vozes. 